\title{
The Lower Limits of Perception of Electrical Currents by Fish.
}

\author{
By
}

H. C. Regnart, M.Sc., F.Z.S.

In recent years attention has been given to the influence of electrical currents as fish deflectors or screens, and experiments have been made to determine the magnitude of the currents which are sufficient to paralyse or act as deterrents to fish which may enter the electrical field, ${ }^{*}$ but in such strong fields the perception of the mechanism by which fish respond to electric currents is masked by the paralysis produced. It seemed possible that experiments with very weak currents might give fuller information concerning the mechanism by which fish respond to electrical stimulus than those made in strong fields. There is also the possibility that weak electric currents may occur in nature to which fish respond, but before exploring that field of phenomena it is necessary to observe the behaviour of fish under the influence of very weak fields. McMillan found that the field (volts per inch) required to paralyse young salmon varied with the resistivity of the water ; thus when the latter was $10,000 \mathrm{ohms}$ per inch cube the paralysing voltage was about $1 \cdot 5$, so that a current density of $\frac{1 \cdot 5}{10^{4}}=0 \cdot 00015$ ampere per square inch was sufficient to cause paralysis, whereas when the resistivity was twelve ohms per inch cube the current required was $\cdot 03$ ampere. In the former case the conductivity of the fish was greater than that of the water, in the latter case less.

In the experiments to be described to determine the lower limit a preliminary investigation was made by applying a current field to freelyswimming fish. When a fish is subjected to such a stimulus it is desirable to measure the current in terms of its density, e.g. amperes per square centimetre, otherwise there can be no adequate standard of comparison of the results of different experiments.

Some initial tests were made, using goldfish (Carassius auratus) maintained in a wooden tank $12 \mathrm{ft}$. long containing fresh water. Into this were fitted vertically two rustless steel plates measuring $30 \times 32 \mathrm{~cm}$. wide which exactly fitted the cross section. The tank was placed in a

* F. O. MeMillan, "Electric Fish Screen," Bulletin of U.S. Bureau of Fisheries in Washington, 1929, Vol. XLIV, 1928, p. 97, with current alternating at 60 periods a second. 
separate darkened room free from all forms of vibration and other disturbances. The plates were arranged at a distance of $100 \mathrm{~cm}$. apart, and using these as electrodes the effect of various intensities of direct currents on small fish about $6 \mathrm{~cm}$. long was investigated. The tank was provided with a glass window and the fish were observed from a distance by means of a large periscope. The water was kept at a constant depth of $20 \mathrm{~cm}$. Responses were obtained on completing the circuit, and occasionally again in a weaker form on breaking the circuit, the latter being a response to the removal of the current stimulus. The nature of the responses varied according to the position of the fish. When orientated so that the anterior end was facing the positive or higher potential plate, completing the circuit caused a rapid convulsion and generally an immediate retreat. When, on the other hand, the anterior end of the fish was facing the negative plate, there was often no response or sometimes a slight twitch although the current was the same as before. The field was unidirectional.

When the fish swam transversely across the field responses were slight and infrequent. Repeated application of the current every few seconds caused the responses of the fish to become weaker and finally to cease altogether. These responses were very definite with a total current of 100 milliamperes (and a potential difference of 15 volts across the plates) which corresponds to a current density of $0 \cdot 00016$ amperes per sq. $\mathrm{cm}$.

When the circuit was completed and then broken rapidly, sharper responses were obtained. While the current was passing steadily for a short time, the fish were not affected. Similar, but definite though weaker, responses were observed on completing the circuit carrying a current of 10 milliamperes (and a potential difference across the plates of 1.5 volts) for which the density was 16 microamperes per sq. $\mathrm{cm}$.

There appeared to be no sharply defined lower limit at which the fish became sensitive, and they were shown to respond to still lower current densities than the above by the following experiment. The tank was brightly illuminated and a narrow band of shadow, about 4 inches wide, cast transversely across the middle. The fish at first sheltered in the shaded area, but presently began to emerge and swim into the lighted region; but on making and breaking several times a current of 3 milliamperes (current density $=5$ microamperes per sq. $\mathrm{cm}$.), the fish could all be caused to retreat and assemble again in the shaded area, but not to exhibit the characteristic sudden responses.

The next experiments were performed in sea-water on marine fish, and the same plates as before were lowered into a large floor pool in the Dove Marine Laboratory at Cullercoats, at a distance of 8 feet apart. The length of this pool was 17 feet, its width 10 feet, and its depth $1 \frac{1}{2}$ feet. Three codling (Gadus callarias), each about a foot long, were limited to swim up and down between the plates by means of string netting, so that the 
available cross section of movement was the same as the area of the plates. With direct current varying between 0.2 and 0.6 amperes (and 2-4 volts potential difference on the circuit including the adjustable resistance, this giving some measure of the initial shock) definite responses were obtained with a pendulum switch set for 0.5 sec. contact, but much better results followed when the current was made by a quick action switch; the pendulum contacts were probably too light. After the response at the make of the current, the fish were not affected while it flowed continuously. The effect was mainly at the make of the current, but occasionally in a weaker form at the break. This indicated that a stabbing switch set to work at short intervals, e.g. half a second, would be more effective as a fish screen than a steady current. As before, the most marked results were obtained when the fish were so orientated that the anterior end was facing the positive plate, with occasionally a slight twitch when facing the negative plate. As in the case of the fish tested in fresh water, the responses to repeated stimulus grew less vigorous until finally no effect was obtained at the make and break of the current. Stronger currents caused more vigorous responses and more tests could be made before the fish ceased to respond. Continued trials carried out over several days adversely affected the fish and made them sluggish and unsuitable for further experiment until they had been rested for some days.

In order to place the fish under more natural conditions the nets were removed. The fish then swam freely about, and when facing the positive plate gave better responses with these currents $(0 \cdot 2-0 \cdot 6$ amps. at from $2-4$ volts). A disadvantage of these experiments was that after a few responses the fish avoided the region between the two plates. All these trials were made under suitable conditions with freedom from ground vibrations and other disturbances, and in a weak light. The responses were recorded with the help of three additional observers and no observations accepted unless agreed to by all. Owing to the fact that in this case when working in the floor pool the current between the plates spread out, the actual current densities were from 15 to 45 microamperes per sq. cm.* Similar experiments were made with alternating currents, using the same fish. Under these circumstances the fish gave a slight response when facing the earth plate, but a more vigorous response was observed when facing the high-tension plate. As before, the best results were obtained at the

* Note by Professor W. M. Thornton :

If the current $i$ is taken as spreading out from small cylindrical electrodes the density midway between them is approximately $i / \pi \mathrm{D} t$, where $\mathrm{D}$ is their distance apart and $t$ the depth of the liquid. With $i=0 \cdot 2$ ampere, $\mathrm{D}=250 \mathrm{~cm} ., t=44 \mathrm{~cm}$., this is equal to 5.8 microamperes. If the current is taken as spreading from strip elements of two flat plates $30 \mathrm{~cm}$. wide and the effect is integrated over their width, the density midway is 19 microamperes. A more complete treatment, assuming the electrodes cylindrical and relatively large (see Pidduck, "Electricity and Magnetism," \$46. See also F. O. McMillan, "Electric Fish Screen," Bulletin of the U.S. Bureau of Fisheries, Vol. XLIV, 1928, Fig. 12, p. 117), gives the estimated values 15 to 45 microamperes per sq. cm. 
make and break of the current. With a current of 3 amperes and the plates 6 feet apart, very violent responses were obtained. Trials were made with currents over the range 0.5 to 3 amperes, corresponding to the current densities 0.000038 to 0.00022 ampere per sq. cm., at the lower of which response was just perceptible.

In order to investigate the threshold values in sea-water more definitely the following method was used. Twelve codling, about 8-10 inches long, were maintained in a healthy condition in a tank with a glass side which measured 4 feet 6 inches deep, 4 feet wide, and 4 feet long. A wooden frame covered with string netting which exactly fitted the tank was. lowered into the water until it was 18 inches from the bottom, and the fish were kept confined in this space with the electrodes arranged at each end and 3 feet 4 inches apart. Under these circumstances, the fish, while swimming freely, were constrained to move more slowly and the sudden twitch due to an electric impulse could be very clearly distinguished. Tests were made at 6 -minute intervals. The responses were very definite at first, but after a time became weaker and the fish finally failed altogether to respond. As the current was increased more responses were obtained before the effect ceased. The fish, after two days during which the tests were carried out, became sluggish and refused to feed, and the tests had to be made at least every other day to give them time to recover from the effects of the repeated stimuli and their confinement in a limited space. Alternating current was used over the range 0.025 to 0.65 ampere, which in this case gave the current density range of 2 to 50 microamperes per sq. cm. In these experiments the fish were tested when facing the positive plate and either in the centre of the field or nearer to it. After a time the fish avoided going near the higher tension plate, and this was proved by reversing the leads on the plates. The fish also would avoid swimming frequently between the plates. As before, three additional observers were employed and only vigorous movements regarded as positive; doubtful cases were taken as negative. These responses were quite distinct from the slower movements made when the current was not passing. They consisted of two movements, (1) a sudden twitch, (2) an immediate change of direction in swimming. A larger number of consecutive readings could not be obtained, as after carrying on the experiments for several weeks the fish as before became sluggish and refused to feed.

Experiments were next undertaken to ascertain if possible the regions which function as receptors of the stimulus of the current field; and as a first attempt the lateral canals with their sense organs were considered. As before codling about 15 inches long were used. Three fish were anæsthetized by the addition of some chloroform to the water and cuts made on both sides of each fish, which severed the supra-opthalmic, suborbital, and hyomandibular branches of the facial nerve, the lateralis 
nerve, and the ventral ramus of the lateralis nerve. These operations eliminated the organs of the lateral canals (and also the skin of the anterior part of the head, since cutting the supra-opthalmic branch of the facial nerve also involved cutting the corresponding associated branch of the trigeminal nerve). The fish were left for a fortnight to recover, and as they had but a few days before been obtained directly from the sea, the recoveries were very rapid. Under similar conditions to those already described, the fish were still found to respond to stimuli of the order of 15-30 microamperes per sq. cm. although they were less sensitive than in the case of unoperated fish. One of the fish with lateral organs eliminated was then subjected to section of the optic nerve on both sides and again the sensitivity to electrical stimuli was not completely eliminated. In all these operative experiments the fish were killed after experiment and the fish after being hardened in spirit dissected to verify the nerve-cutting work. These experiments made it probable that other areas of the fish such as gills were involved in the perception of these electrical stimuli, but the marked reduction in the strength of the response caused by eliminating the lateral organs made it certain that they played a large part in the response to electrical currents. This is possibly due to the relatively high electrical conductivity of the mucus in the canals, but this point requires further investigation.

As a further proof of the role played by the lateral organs as electrical receptors the following experiments were made which show that these organs may have influence in the perception of the direction of the source of an electrical current in the sea. On the application of an electric field to a normal freely swimming fish, the direction of movement of response was found to depend on the orientation of the fish with respect to the field. Thus, when swimming towards the higher tension plate and inclined to it at a slight angle, the response was generally such that it swam away from the plate in a direction either to left or right which removed it most rapidly from that region.

Two fishes were then operated on by in one cutting the supra-opthalmic branch of the facial nerve on the right side and in the other the corresponding nerve on the left side, and on recovery they behaved like normal fish in their directional movements with respect to the positive plate. Finally, two fish were each subjected to section of all the nerves to the lateral canals on the right side; these fish were then found to give a strong twitch to the left on stimulation whatever their position in the field might be when facing the higher potential plate. These results were obtained over a range of 300 tests and the response to the left was 100 per cent. Next two fresh fish were taken and all the lateral organs on the left side eliminated in each case. These fish gave a twitch to the right over a corresponding series of tests. 
These experiments indicated that the lateral canals with their mucous secretions and the organs associated with them may have considerable importance as receptors for directional purposes, and support the suggestion that the greater development of these organs in certain deep-sea fish is for this purpose.*

It should be emphasized that the above experiments were performed upon fish which were exposed to light and other unnatural environmental conditions and that due to confinement and diet they could not be comparable in vigour and sensitiveness with those in a free state; but even under such unfavourable conditions the results obtained prove that fish are extremely sensitive to electric currents.

The above results may be summarised as follows :

1. Goldfish had a lower sensitivity threshold at about 5 microamperes per sq. cm. direct current.

2. With Codling in a shallow floor pool the threshold was about 15 microamperes per sq. cm. direct current.

3. In a large wall tank their threshold value was about 2 microamperes per sq. cm. alternating current.

4. The lateral organs are proved to play a most important part in the perception of electric stimulus. Section of the nerves of those on the right-hand side caused the fish to throw to the left, and vice versa.

The early experiments were carried out in an annex of the Electrical Engineering Laboratory at Armstrong College; the experiments on sea fish were made by kind permission at the Dove Marine Laboratory, at Cullercoats.

I am greatly indebted to Professor W. M. Thornton for suggesting the research and for his constant help and advice.

*Vide “Electrical Perception by Deep Sea Fish," W. M. Thornton. University of Durham Philosophical Society. Proc., Vol. 8, Part 4. 